\title{
Covariant and Contravariant Symbols of Operators on $l^{2}(\mathbb{Z})$
}

\author{
Abdelhamid S. Elmabrok \\ Department of Mathematics, Faculty of Science, University of Benghazi, Benghazi, Libya
}

\begin{abstract}
Article Info
Keywords: Bounded, Compact and finite rank operators, Covariant and contravariant symbols of operators, Wavelet transformation 2010 AMS: 43A70, 47F05

Received: 10 April 2020

Accepted: 18 August 2020

Available online: 15 December 2020
\end{abstract}

\begin{abstract}
In this paper, we investigate covariant and contravariant symbols of operators generated by a representation of the integer group $\mathbb{Z}$. Then we describe some properties (Existence, Uniqueness, Boundedness, Compactnessi and Finite rank) of these operators and reformulated some know results in terms of wavelet transform (covariant and contravariant symbols).
\end{abstract}

\section{Introduction}

The notion of covariant and contravariant symbols of operators was introduced by Berezin in 1972 [1], as a generalization of a Wick and anti-Wick operator symbols [2]. Then a general theory of quantization was developed by F. A. Berezin in [3]. The construction of wavelet transform as covariant and contravariant symbols was realized with wavelets in Hilbert spaces [4]. Recently, in 2014 V. Kisil in his paper [5, Sec 4.2] studied Berezin covariant symbols as a special case of the covariant transform. Also, he applied wavelets on operator algebras by means of symbols of operators [6], which is an extension of the Berezin calculus. The purpose of the present paper is to describe some properties (existence, uniqueness, boundedness and compactness) of operators which have covariant and contravariant symbols and reformulated some know results on these operators.

The paper outline is as follows: In the second Section, we collects preliminary information from other works, which will be used here. In particular, the concepts of covariant and contravariant symbols of operators. In the third Section, we describe some proprieties of covariant and contravariant symbols of operators which generated by the representation of the integer group. Then, we reformulate some know results on existence, uniqueness, boundedness and compactness of linear operators in terms of wavelet transform. The final Section offers summary of our observations which lead to new directions for further research.

\section{Preliminaries}

In this section we present some fundamental concepts and known results on boundedness and compactness of linear operators in Hilbert spaces and wavelet transform on groups. We denoted by $B(\mathscr{H})$ the sets of all bounded linear operator $A$ on Hilbert space $\mathscr{H}$. Let $\mathbb{G}$ be a group with a left Haar measure $d \mu$ and let $\pi$ be a unitary irreducible representation of a group $\mathbb{G}$ by operators $\pi_{g}, g \in \mathbb{G}$ in a Hilbert space $\mathscr{H}$.

Definition 2.1. [6] Let $\psi_{0}$ be a fixed vector in a space $\mathscr{H}$, it is called a vacuum vector (mother wavelet). Then the set of vectors $\psi_{g}=\pi(g) \psi_{0}$ for $g \in \mathbb{G}$ is called a family of coherent states (wavelets). We define wavelet transform as a mapping $\mathscr{W}$ 
from the Hilbert space to a space of functions over a group $\mathbb{G}$ via its representational coefficients $\mathscr{W}: \mathscr{H} \rightarrow L(\mathbb{G}): v \mapsto \hat{v}(g)$, by

$$
\hat{v}(g)=\left\langle\pi\left(g^{-1}\right) v, \psi_{0}\right\rangle=\left\langle v, \pi(g) \psi_{0}\right\rangle=\left\langle v, \psi_{g}\right\rangle .
$$

The wavelet transform $\mathscr{W}$ is a continuous linear mapping and the image of a vector is a bounded continuous function on $\mathbb{G}$. The linear space of all such images is denoted by $W(\mathbb{G})$.

Definition 2.2. [6] The inverse wavelet transform is a mapping $\mathscr{M}: L_{1}(\mathbb{G}) \rightarrow \mathscr{H}: \hat{v}(g) \mapsto \mathscr{M}[\hat{v}(g)]$ given by the formula:

$$
\mathscr{M}[\hat{v}(g)]=\int_{\mathbb{G}} \hat{v}(g) \pi(g) d \mu(g) \psi_{0}=\int_{\mathbb{G}} \hat{v}(g) \psi_{g} d \mu(g),
$$

where the integral expresses an operator acting on vector $\psi_{0}$.

An important observation [6] is that, two representations for groups $\mathbb{G}$ and $\mathbb{G} \times \mathbb{G}$ were defined correspondingly in the space $B(\mathscr{H})$ of bounded linear operators $\mathscr{H} \rightarrow \mathscr{H}$ as follows:

$$
\begin{gathered}
\hat{\pi}: \mathbb{G} \rightarrow B(B(\mathscr{H})): A \longmapsto \pi(g)^{-1} A \pi(g) \\
\breve{\pi}: \mathbb{G} \times \mathbb{G} \rightarrow B(B(\mathscr{H})): A \longmapsto \pi\left(g_{1}\right)^{-1} A \pi\left(g_{2}\right)
\end{gathered}
$$

where $A \in B(\mathscr{H})$.

Let there be selected a vacuum vector $h_{0} \in \mathscr{H}$ and a test functional $l_{0} \in \mathscr{H}^{*}$ for $\pi$. Then there are canonically associated vacuum vector $p_{0} \in B(\mathscr{H})$ and test functional $f_{0} \in B^{*}(\mathscr{H})$ defined as follows:

$$
\begin{gathered}
p_{0}: \mathscr{H} \longrightarrow \mathscr{H}: h \longmapsto p_{0} h=\left\langle h, l_{0}\right\rangle h_{0} ; \\
f_{0}: B(\mathscr{H}) \longrightarrow \mathbb{C}: A \longmapsto\left\langle A h_{0}, l_{0}\right\rangle .
\end{gathered}
$$

They define the following coherent states and transformations of the test functional

$$
\begin{gathered}
p_{g}=\hat{\pi}(g) p_{0}=\left\langle\cdot, l_{g}\right\rangle h_{g}, \quad p_{\left(g_{1}, g_{2}\right)}=\breve{\pi}\left(g_{1}, g_{2}\right) p_{0}=\left\langle\cdot, l_{g_{1}}\right\rangle h_{g_{2}}, \\
f_{g}=\hat{\pi}^{*}(g) f_{0}=\left\langle\cdot h_{g}, l_{g}\right\rangle, \quad f_{\left(g_{1}, g_{2}\right)}=\breve{\pi}^{*}\left(g_{1}, g_{2}\right) f_{0}=\left\langle\cdot h_{g_{1}}, l_{g_{2}}\right\rangle,
\end{gathered}
$$

where as usual we denote $h_{g}=\pi(g) h_{0}, l_{g}=\pi^{*}(g) l_{0}$.

Definition 2.3. [6] The covariant symbol $\tilde{a}(g)\left(\tilde{a}\left(g_{1}, g_{2}\right)\right)$ of an operator A acting on a Hilbert space $\mathscr{H}$ defined by $h_{0} \in \mathscr{H}$ and $l_{0} \in \mathscr{H}^{*}$ is its wavelet transform with respect to the representation $\hat{\pi}(g),\left(\breve{\pi}\left(g_{1}, g_{2}\right)\right)$ respectively and the functional $f_{0}$, they are defined by the formulas

$$
\begin{gathered}
\tilde{a}(g)=\left(\hat{\pi}(g) A, f_{0}\right)=\left\langle\pi(g)^{-1} A \pi(g) h_{0}, l_{0}\right\rangle=\left\langle A h_{g}, l_{g}\right\rangle, \\
\tilde{a}\left(g_{1}, g_{2}\right)=\left(\breve{\pi}\left(g_{1}, g_{2}\right) A, f_{0}\right)=\left\langle\pi\left(g_{1}\right)^{-1} A \pi\left(g_{2}\right) h_{0}, l_{0}\right\rangle=\left\langle A h_{g_{2}}, l_{g_{1}}\right\rangle .
\end{gathered}
$$

Definition 2.4. [6] The contravariant symbol of an operator $A$ is a function $a(g)$ and $\left(a\left(g_{1}, g_{2}\right)\right)$ such that $A$ is the inverse wavelet transform of $a(g), a\left(g_{1}, g_{2}\right)$ correspondingly with respect to $\hat{\pi}(g), \breve{\pi}\left(g_{1}, g_{2}\right)$, i.e.

$$
\begin{aligned}
A= & \int_{G} a(g) \hat{\pi}(g) p_{0} d \mu(g)=\int_{G} a(g) p_{g} d \mu(g) . \\
A & =\int_{\mathbb{G}} \int_{\mathbb{G}} a\left(g_{1}, g_{2}\right) \breve{\pi}\left(g_{1}, g_{2}\right) p_{0} d \mu\left(g_{1}\right) d \mu\left(g_{2}\right) \\
& =\int_{\mathbb{G}} \int_{\mathbb{G}} a\left(g_{1}, g_{2}\right) p_{\left(g_{1}, g_{2}\right)} d \mu\left(g_{1}\right) d \mu\left(g_{2}\right),
\end{aligned}
$$

where the integral is defined in the weak sense. 
Now, we turn to the separable Hilbert space $\mathscr{H}$ isomorphic to $l^{2}(\mathbb{Z})$ which is the space of all square-summable complex sequences on $\mathbb{Z}$. Formally

$$
l^{2}(\mathbb{Z})=\left\{x(n), n \in \mathbb{Z}: x(n) \in \mathbb{C} \quad \text { and } \quad \sum_{n \in \mathbb{Z}}|x(n)|^{2}<\infty\right\},
$$

with the inner product and the norm, respectively,

$$
\langle x, y\rangle=\sum_{n \in \mathbb{Z}} x(n) \overline{y(n)}, \quad\|x\|=\left(\sum_{n \in \mathbb{Z}}|x(n)|^{2}\right)^{\frac{1}{2}} .
$$

Let $X^{\prime}$ be a dual space (conjugate space), which is a normed vector space of all bounded linear functional from a normed space $X$ into the field $\mathbb{C}$, with norm

$$
\|f\|=\sup \{|f(x)|:\|x\| \leq 1\} .
$$

Also, if $X^{\prime}$ is the dual of a Banach space $X$. Then for all $x \in X$

$$
\|x\|=\max \left\{|f(x)|: f \in X^{\prime},\|f\|=1\right\}
$$

Definition 2.5. Let $F(S, X)$ is denoted to the collection of functions $S \rightarrow X$ for set $S$ and a vector space $X$ over $\mathbb{C}$. The support of $f \in F(S, X)$ is

$$
\operatorname{supp}(f):=\{s \in S: f(s) \neq 0\}=f^{-1}(X \backslash\{0\}) .
$$

The collection of functions $S \rightarrow X$ having finite support is denoted

$$
\mathscr{F}_{00}(S, X):=\{f \in F(S, X): \operatorname{supp}(f) \subset \subset S\},
$$

where $\subset \subset$ denoted to a subset of finite cardinality. Also,

$$
F(S)=F(S, \mathbb{C}) \quad \text { and } \quad \mathscr{F}_{00}(S)=\mathscr{F}_{00}(S, \mathbb{C}) .
$$

Proposition 2.6. [7, p.98] The necessary and sufficient condition that there exist a bounded linear operator A defined on $\mathscr{H}$ such that $\left\langle A e_{n}, e_{m}\right\rangle=a_{m n}$, is that, for any finite $p$ and $q$ and for arbitrary $\alpha_{1}, \alpha_{2}, \ldots, \alpha_{p} ; \beta_{1}, \beta_{2}, \ldots, \beta_{q}$, the inequality

$$
\left|\sum_{m}^{p} \sum_{n}^{q} a_{m n} \alpha_{m} \bar{\beta}_{n}\right| \leq M \sqrt{\sum_{m}^{p}\left|\alpha_{m}\right|^{2}} \sqrt{\sum_{n}^{q}\left|\beta_{n}\right|^{2}},
$$

holds, $M$ being a fixed number.

Proposition 2.7. [8] A bounded operator $A \in B(\mathscr{H})$ is compact if and only if satisfies:

$$
\lim _{n \rightarrow \infty} A e_{n}=0
$$

for each orthonormal basis for $\mathscr{H}$.

Proposition 2.8. [9, p.91] Let $A$ be a finite rank linear operator in $\mathscr{H}$ into itself, then $A$ is compact.

Definition 2.9. [10, p.442] Let $\mathscr{H}$ be a Hilbert space and let $\left\{P_{m}\right\}$ be a resolution of the identity defined on $\mathscr{H}$. Further, let $\left\{\lambda_{m}\right\}$ be a sequence of scalars. A transformation of the form

$$
A v=\sum_{m=1}^{\infty} \lambda_{m} P_{m} v, v \in D_{A}
$$

where

$$
D_{A}=\left\{v \in \mathscr{H}: \lim _{N \rightarrow \infty} \sum_{m}^{N} \lambda_{m} P_{m} v, \text { exists }\right\}
$$

is said to be a weighted sum of projections.

Theorem 2.10. [10] Let A be a compact normal operator on a Hilbert space $\mathscr{H}$. Then there is a resolution of the identity $\left\{P_{m}\right\}$ and a sequence of complex numbers $\left\{\lambda_{m}\right\}$ such that $A=\sum_{m} \lambda_{m} P_{m}$, where the convergence is in terms of the uniform operator norm topology. 


\section{Covariant and contravariant symbols of operators generated by representation of the integer group}

One goal of this paper is to describe some proprieties of covariant and contravariant symbols of operators which generated by the representation of the integer group. We can reformulate some know results on existence, uniqueness, boundedness and compactness of linear operators in terms of covariant and contravariant symbols (wavelet transform).

\subsection{Wavelet transforms for the integer group in $l^{2}(\mathbb{Z})$ space}

The subject of wavelet transform has arisen many times in many applied areas and we are not able to give a comprehensive history and proper credit. One could mention important books [11, 12]. In the first part of this section, we look for wavelet transformation in a separable Hilbert space $l^{2}(\mathbb{Z})$ ) with an orthonormal basis $\left\{e_{k}\right\}, k \in \mathbb{Z}$. Then the group $\mathbb{Z}$ of integers has a unitary representation $\pi$ on $l^{2}(\mathbb{Z})$, which is defined on the base as follows

$$
\pi(m) e_{k}=e_{k+m}, m \in \mathbb{Z}
$$

The adjoint representation is

$$
\pi^{*}(m) e_{k}=e_{k-m}, m \in \mathbb{Z}
$$

Hence, $e_{0}$ could be taken as a vacuum vector and test functional. Therefore, by Equation (2.1) the wavelet transform with a vacuum vector $\psi_{0}=e_{0}$ is

$$
\mathscr{W}(v)=\hat{v}(m)=\left\langle v, \pi(m) e_{0}\right\rangle=\left\langle v, e_{m}\right\rangle .
$$

and by Equation (2.2) the inverse wavelet transform is

$$
\mathscr{M}[\hat{v}(m)]=\sum_{\mathbb{Z}} \hat{v}(m) \pi(m) e_{0}=\sum_{-\infty}^{\infty} \hat{v}(m) e_{m}
$$

This is the Fourier series.

Inspired by the corresponding propositions, [13, Sec 4.1], we now equivalently reformulate the following:

Remark 3.1. The left regular representation $\Lambda(m)$ of the group $\mathbb{Z}$ is the unitary representation by left shifts in the space $l^{2}(\mathbb{Z})$ by

$$
\Lambda(m): v(m) \longrightarrow v(-m+n) .
$$

Proposition 3.2. The wavelet transform $\mathscr{W}$ intertwines $\pi$ and the left regular representation $\Lambda$ (3.3) of $\mathbb{Z}$ :

$$
\mathscr{W} \pi(m)=\Lambda(m) \mathscr{W}
$$

Proof. By Equations (3.1) and (3.3). We have

$$
\begin{aligned}
{[\mathscr{W} \pi(m) v](n) } & =\left\langle\pi(m) v, \pi(n) e_{0}\right\rangle, \\
& =\left\langle v, \pi^{*}(m) \pi(n) e_{0}\right\rangle, \\
& =\left\langle v, \pi^{*}(m) e_{n}\right\rangle, \\
& =\left\langle v, e_{(-m+n)}\right\rangle, \\
& =[\mathscr{W} v](-m+n), \\
& =\Lambda(m)[\mathscr{W} v](n) .
\end{aligned}
$$

Corollary 3.3. The function space $W(\mathbb{Z})$ is invariant under the representation $\Lambda$ of $\mathbb{Z}$.

Proposition 3.4. The inverse wavelet transform $\mathscr{M}$ intertwines $\Lambda$ on $L_{2}(\mathbb{Z})$ and $\pi$ on $\mathscr{H}$ :

$$
\mathscr{M} \Lambda(m)=\pi(m) \mathscr{M} .
$$


Proof. By Equations (3.2) and (3.3). We have

$$
\begin{aligned}
\mathscr{M}[\Lambda(m) \hat{v}(n)] & =\mathscr{M}[\hat{v}(-m+n)], \\
& =\sum_{n} \hat{v}(-m+n) \pi(n) e_{0}, \\
& =\sum_{n} \hat{v}(-m+n) e_{n}, \\
& =\sum_{k} \hat{v}(k) e_{m+k}, \\
& =\sum_{k} \hat{v}(k) \pi(m) e_{k}, \\
& =\pi(m) \sum_{k} \hat{v}(k) e_{k}, \\
& =\pi(m) \mathscr{M}[\hat{v}(k)],
\end{aligned}
$$

where, $k=n-m$.

Corollary 3.5. The image $\mathscr{M}\left(L_{1}(\mathbb{Z})\right) \subset \mathscr{H}$ of subspace under the inverse wavelet transform $\mathscr{M}$ is invariant under the representation $\pi$.

Proposition 3.6. The image $\mathscr{W}(\mathbb{Z})$ of the wavelet transform $\mathscr{W}$ has a reproducing kernel $K(m, n)=\left\langle w_{m}, w_{n}\right\rangle$. The reproducing formula is in fact a Discrete convolution:

$$
\hat{v}(n)=\sum_{m \in \mathbb{Z}} k(n, m) \hat{v}(m)=\sum_{m \in \mathbb{Z}} \hat{w}_{0}(n-m) \hat{v}(m) .
$$

with a wavelet transform of the vacuum vector $\hat{w}_{0}(n)=\left\langle w_{0}, \pi(n) w_{0}\right\rangle$.

Proof. By Equation (3.1) and since $\pi$ is an irreducible square integrable representation defined by the same admissible vector $w_{0}([13, \operatorname{Sec} 8.2])$ we have

$$
\begin{aligned}
\hat{v}(n) & =\left\langle\pi(-n) v, w_{0}\right\rangle, \\
& =\sum_{m \in \mathbb{Z}}\left\langle\pi(-k) \pi(-n) v, w_{0}\right\rangle \overline{\left\langle\pi(-k) w_{0}, w_{0}\right\rangle}, \\
& =\sum_{m \in \mathbb{Z}}\left\langle\pi(-(k+n)) v, w_{0}\right\rangle\left\langle\pi(k) w_{0}, w_{0}\right\rangle, \\
& =\sum_{m \in \mathbb{Z}} \hat{v}(n+k) \hat{w}_{0}(-k), \\
& =\sum_{m \in \mathbb{Z}} \hat{v}(m) \hat{w}_{0}(n-m) .
\end{aligned}
$$

\subsection{Covariant and contravariant symbols}

Berezin symbols and coherent states are a useful tool in quantum theory and have a lot of essentially diferent definitions [14]-[16]. In particular, they were described by Berezin, concerning so-called covariant and contravariant (or Wick and anti-Wick) symbols of operators (see, for example, [17]-[19]). In the second part of this section as the first applications, we describe the covariant and contravariant symbols of operators which realizes the unitary irreducible representations of $\mathbb{Z}$ on Hilbert spaces $l^{2}(\mathbb{Z})$. By Equations (2.3) and (2.4) the covariant symbol $\tilde{a}(m), \tilde{a}(m, n)$ of an operator $A \in l^{2}(\mathbb{Z})$ is its wavelet transform with respect to the representation $\hat{\pi}(m), \breve{\pi}(m, n)$, i.e.

$$
\begin{aligned}
\tilde{a}(m) & =\left(\hat{\pi}(m) A, e_{0}\right)=\left\langle\pi(m)^{-1} A \pi(m) e_{0}, e_{0}\right\rangle \\
& =\left\langle A e_{m}, e_{m}\right\rangle=a_{m m}, \\
\tilde{a}(m, n) & =\left(\breve{\pi}(m, n) A, e_{0}\right)=\left\langle\pi(m)^{-1} A \pi(n) e_{0}, e_{0}\right\rangle \\
& =\left\langle A e_{n}, e_{m}\right\rangle=a_{m n},
\end{aligned}
$$

where $a_{m n}$ is a matrix representation in orthonormal basis $e_{k}$.

Now, by Equation (2.5) A is the inverse wavelet transform of $a(m)$ with respect to $\hat{\pi}(m)$, where the function $a(m)$ is the contravariant symbol of an operator A, i.e.

$$
A=\sum_{m \in \mathbb{Z}} a(m) \hat{\pi}(m) p_{0}=\sum_{m \in \mathbb{Z}} a(m) p_{m},
$$




$$
A e_{k}=\sum_{m \in \mathbb{Z}} a(m) p_{m} e_{k}=\sum_{m \in \mathbb{Z}} a(m) e_{m} \cdot \delta_{k m}=a(k) e_{k},
$$

where $\delta_{k m}$ is the Kronecker delta. Similarly, by (2.6) the inverse wavelet transform of $a(m, n)$ with respect to $\breve{\pi}(m, n)$ is

$$
\begin{gathered}
A=\sum_{n \in \mathbb{Z}} \sum_{m \in \mathbb{Z}} a(m, n) \breve{\pi}(m, n) p_{0}=\sum_{n \in \mathbb{Z}} \sum_{m \in \mathbb{Z}} a(m, n) p_{(m, n)} \\
A e_{k}=\sum_{n \in \mathbb{Z}} \sum_{m \in \mathbb{Z}} a(m, n) p_{(m, n)} e_{k}=\sum_{n \in \mathbb{Z}} a(k, n) e_{n} .
\end{gathered}
$$

Remark 3.7. (i) The coherent states $p_{m}$ and $p_{(m, n)}$ are a rank-one operators,

(ii) The formula (3.5) yields a representation of $A$ as an infinite matrix.

\subsection{Some proprieties of covariant and contravariant symbols}

The Berezin symbol of an operator provides important information about the operators, in particular, that, the Berezin symbol uniquely determines the operator (i.e., $A=0$ if and only if $\tilde{A}=0$ ), (see, for example, [20]-[24]). In the third part of this section we will classify some proprieties of covariant and contravariant symbols of operators in $B\left(l_{2}(\mathbb{Z})\right)$. Also, we will discuss further questions and reformulate some know results on existence, uniqueness, boundedness and compactness of these operators in terms of covariant and contravaraint symbols of operators: First, does every operator on $l^{2}(\mathbb{Z})$ have covariant symbols (3.4) and (3.5)? If yes, is it unique? If not, how to characterize operators which do have? This question is answered by the following proposition.

Proposition 3.8. For every bounded operator $A$ on $l^{2}(\mathbb{Z})$ ), there exists a unique covariant symbol given by formula (3.5).

Proof. Let $A$ be a bounded operator on $l^{2}(\mathbb{Z})$. Since $l^{2}(\mathbb{Z})$ contains an orthonormal basis $\left\{e_{n}\right\}$ (they span $l^{2}(\mathbb{Z})$ ). Also the set

$$
\left\{e_{n} \in l^{2}(\mathbb{Z}): A e_{n}=\sum_{m} a_{m n} e_{m}=b_{m} \in l^{2}(\mathbb{Z})\right\}
$$

which is subset of $D_{A}$ domain of $A$. Therefore, any bounded operator on $l^{2}(\mathbb{Z})$ can be represent as a matrix $a_{m n}=\left\langle A e_{n}, e_{m}\right\rangle=$ $\tilde{a}(m, n)$, which is (3.5). To prove uniqueness of covariant symbols $\tilde{a}(m, n)$. From Riesz representation theorem on $l^{2}(\mathbb{Z})$, one simply notes that, there is one element $A e_{n} \in l^{2}(\mathbb{Z})$, such that $\left\langle A e_{n}, e_{m}\right\rangle=\tilde{a}(m, n)$. Hence, $\tilde{a}(m, n)$ is unique.

Remark 3.9. The formula (3.4) is a special case of (3.5) where $m=n$.

The second question was about the contravariant symbol. Does any operator on $l^{2}(\mathbb{Z})$ have contravariant symbols (3.6) and (3.7)? If yes, is it unique? If not, how to characterize operator which do have?

Not every operator $A$ on $l^{2}(\mathbb{Z})$ has a contravariant symbol satisfying (3.6). For example, let $A$ be the operator of multiplication by $t$ on $L^{2}[0,1]$ defined by $(A v)(t)=t v(t)$. $A$ is a self-adjoint, bounded operator and has no eigenvalue. Therefore it does not have contravariant symbol $a(m)$ which satisfies (3.6).

Proposition 3.10. For every compact normal operator $A$ on $l^{2}(\mathbb{Z})$, there exists a unique contravariant symbol a $\left.(m)\right)$ satisfying formula (3.6).

Proof. Let $A$ be a compact normal operator on $l^{2}(\mathbb{Z})$. Then by Theorem 2.10 there is a resolution of the identity $\left\{P_{m}\right\}$ where $P_{m}$ is an orthogonal projection and a measurable complex-valued function $a(m)$ on $\mathbb{Z}$ (weighted function) such that the operator $A$ can be expressed as weighted sums (2.9)

$$
A=\sum_{m \in \mathbb{Z}} a(m) P_{m}
$$

which is (3.6). It is easily to prove uniqueness of the contravariant symbol $a(m)$. We omit it here.

Remark 3.11. Formula (3.7) in the second question still needs more discussion.

The third question, how to see from a covariant or contravariant symbol of operator if it is finite rank?

Not all operator on $l^{2}(\mathbb{Z})$ which have a covariant symbol satisfying (3.5) are of finite rank. For example, let $A$ be the identity operator $I$ which has the covariant symbol $I(m, n)=\left\langle I_{n}, e_{m}\right\rangle=\delta_{m n}$. But $I e_{n}=e_{n}$. Therefore $\operatorname{rak}(I)=\operatorname{dim}(\operatorname{Im}(I))=\infty$. Then $I$ is not finite rank operator.

Proposition 3.12. If the covariant symbol which satisfy (2.6) equal zero for any $m<m_{1}$ or $m>m_{2}$ and for all $n$, then $A$ is a finite rank operator on $l^{2}(\mathbb{Z})$. 
Proof. Let the covariant symbol $\tilde{a}(m, n)=\left\langle A e_{n}, e_{m}\right\rangle=a_{m n}=0$, for any $m<m_{1}$ or $m>m_{2}$ and for all $n$. Since $A$ define on $l^{2}(\mathbb{Z})$. Then there exists a complete orthonormal basis $e_{m} \in l^{2}(\mathbb{Z})$ such that the range of $A$ is spanned by $e_{m_{1}}, e_{m_{1}+1}, \ldots ., e_{m_{2}}$ So $A$ has a finite rank.

Also, not all operators on $l^{2}(\mathbb{Z})$ which have a contravariant symbol satisfying (3.7) are of finite rank. For example, let $A$ be the identity operator $I$ which has the contravariant symbol $\delta_{m n}$ such that

$$
I v=\sum_{m} \sum_{n} \delta_{m n} P_{m n} v=\sum_{m}\left\langle v, e_{m}\right\rangle e_{m}
$$

But $I v=v$. Therefore $\operatorname{rak}(I)=\operatorname{dim}(\operatorname{Im}(I))=\infty$. Then $I$ is not a finite rank operator.

Proposition 3.13. If an operator $A$ on $l^{2}(\mathbb{Z})$ has the contravariant symbol $a(m, n)$ which satisfies $(3.7)$ such that a $(m, n)=0$ for any $m<m_{1}$ or $m<m_{2}$ and for all $n$, then $A$ is a finite rank operator.

Proof. Let $A$ on $l^{2}(\mathbb{Z})$ have the contravariant symbol $a(m, n)$ satisfying (3.7) such that $a(m, n)=0$ for any $m<m_{1}$ or $m<m_{2}$ and for all $n$. Then there exists a complete orthonormal basis $\left\{e_{m}\right\} \in l^{2}(\mathbb{Z})$ such that the range of $A$ is spanned by $e_{m_{1}}, e_{m_{1}+1}, \ldots, e_{m_{2}}$. Now by (3.7)

$$
A=\sum_{m=m_{1}}^{m_{2}} \sum_{n \in \mathbb{Z}} a(m, n)\left\langle., e_{n}\right\rangle e_{m}
$$

Then $A$ is a finite rank operator.

Proposition 3.14. A bounded operator $A \in B(\mathscr{H})$ is compact if and only if the covariant symbol $a_{m n}$ for a fixed $n$ makes a $l^{2}$ function on $\mathbb{Z}$ such that its norm tends to 0 as $n \longrightarrow \infty$.

Proof. Let $A$ be a compact linear operator from $l^{2}(\mathbb{Z})$ into itself and $\left(e_{n}\right)$ a complete orthonormal set in $l^{2}(\mathbb{Z})$, therefore $A e_{n}=\sum_{m \in \mathbb{Z}} a_{m n} e_{m}$.

Then by proposition 2.7 Equation (2.8).

$$
\begin{aligned}
& \Leftrightarrow \quad \lim _{n \rightarrow \infty} A e_{n}=0, \\
& \Leftrightarrow \quad \lim _{n \rightarrow \infty} \sum_{m \in \mathbb{Z}} a_{m n} e_{m}=0, \\
& \Leftrightarrow \quad \lim _{n \rightarrow \infty} \sum_{m \in \mathbb{Z}}\left|a_{m n}\right|^{2}=0, \\
& \Leftrightarrow \quad \lim _{n \rightarrow \infty}\left\|a_{n}\right\|=0,
\end{aligned}
$$

where $a_{n}=a_{m n}$, for a fixed $n$, with the norm $\left\|a_{n}\right\|=\left(\sum_{m \in \mathbb{Z}}\left|a_{m n}\right|^{2}\right)^{\frac{1}{2}}$.

Proposition 3.15. Let for an operator $A$, there exist a basis $e_{m}$ such that the covariant symbol $a_{m n}=0$, for any $m<m_{1}$ or $m>m_{2}$ and for all $n$, then $A$ is compact.

Proof. For an operator $A$ let there exist a basis $e_{m}$ such that the covariant symbol $a_{m n} \neq 0$ only for $m_{1} \leq m \leq m_{2}$, then the range of $A$ is spanned by $e_{m_{1}}, e_{m_{1}+1}, \ldots, e_{m_{2}}$. Therefore $A$ has finite rank. Finally, by proposition $2.8 A$ is compact.

Proposition 3.16. Let $A$ be an operator acting on a Hilbert space $\mathscr{H}$ and the covariant symbol $a_{m n}$ of A belong to the Banach space $\mathscr{F}_{00}^{\prime}(\mathbb{Z} \times \mathbb{Z})$ with norm

$$
\left\|a_{m n}\right\|:=\sup \left\{\left|\left\langle a_{m n}, l_{m n}\right\rangle\right|: l_{m n} \in \mathscr{F}_{00}(\mathbb{Z} \times \mathbb{Z}),\left\|l_{m n}\right\|_{2}=1\right\} .
$$

Then the operator $A$ is bounded if and only if the covariant symbol $a_{m n}$ is bounded in $\mathscr{F}_{00}^{\prime}(\mathbb{Z} \times \mathbb{Z})$.

Proof. Let $A$ be an operator acting on a Hilbert space $\mathscr{H}$. Let its covariant symbol $a_{m n}$ be bounded in a Banach space $\mathscr{F}_{00}^{\prime}(\mathbb{Z} \times \mathbb{Z})$ with norm $\left\|a_{m n}\right\|$ and

$$
l_{m n}=\alpha \otimes \bar{\beta} \in \mathscr{F}_{00}(\mathbb{Z} \times \mathbb{Z}) \cong \mathscr{F}_{00}(\mathbb{Z}) \otimes \mathscr{F}_{00}(\mathbb{Z}),
$$

then by proposition 2.6 and Equation (3.8), we have

$$
\begin{aligned}
\left|\left\langle a_{m n}, l_{m n}\right\rangle\right| & \leq\left\|a_{m n}\right\|\left\|l_{m n}\right\|_{2} \\
\left|\left\langle a_{m n}, \alpha \otimes \bar{\beta}\right\rangle\right| & \leq\left\|a_{m n}\right\|\|\alpha \otimes \beta\|_{2} \\
\left|\sum_{m, n} a_{m n} \alpha_{m} \overline{\beta_{n}}\right| & \leq M\|\alpha\|_{2}\|\beta\|_{2} .
\end{aligned}
$$


Thus, $A$ is bounded.

Conversely, let $A$ be a bounded linear operator acting on a Hilbert space $\mathscr{H}$ and $a_{m n}$ its covariant symbol in a Banach space $\mathscr{F}_{00}^{\prime}(\mathbb{Z} \times \mathbb{Z})$ with norm (3.8), then by necessary condition of proposition $2.6 a_{m n}$ satisfies the inequality (2.7), i.e

$$
\left|\sum_{m}^{p} \sum_{n}^{q} a_{m n} \alpha_{m} \bar{\beta}_{n}\right| \leq M\|\alpha\|_{2}\|\beta\|_{2},
$$

therefore by equation (3.8)

$$
\begin{aligned}
\left|\left\langle a_{m n}, \alpha \otimes \bar{\beta}\right\rangle\right| & \leq M\|\alpha\|_{2}\|\beta\|_{2} \\
\left|\left\langle a_{m n}, l_{m n}\right\rangle\right| & \leq M\left\|l_{m n}\right\|_{2} .
\end{aligned}
$$

Then $a_{m n}$ is bounded in $\mathscr{F}_{00}^{\prime}(\mathbb{Z} \times \mathbb{Z})$.

Proposition 3.17. The mapping $\sigma: A \longmapsto \sigma_{A}(m, n)$ of operators to their covariant symbols is an algebra homomorphism from the algebra of operators on $\mathscr{H}$ to the algebra of infinite matrices on $W(\mathbb{Z})$, i.e.

$$
\sigma_{A_{1} A_{2}}(m, k)=\sum_{n \in \mathbb{Z}} \sigma_{A_{1}}(m, n) \sigma_{A_{2}}(n, k)
$$

Proof. By (3.5), we have

$$
\begin{aligned}
\sigma_{A_{1} A_{2}}(m, k) & =\left\langle A_{1} A_{2} e_{m}, e_{n}\right\rangle \\
& =\left\langle A_{1} A_{2} \pi(m) h_{0}, \pi(n) l_{0}\right\rangle \\
& =\left\langle\pi(m) A_{1} A_{2} \pi^{-1}(n) h_{0}, l_{0}\right\rangle \\
& =\left\langle A_{2} \pi^{-1}(n) h_{0}, A_{1}^{*} \pi^{*}(m) l_{0}\right\rangle, \\
& =\sum_{n}\left\langle\pi(k) A_{2} \pi^{-1}(n) h_{0}, l_{0}\right\rangle,\left\langle\pi(k) h_{0}, A_{1}^{*} \pi^{*}(m) l_{0}\right\rangle, \\
& =\sum_{n}\left\langle\pi(m) A_{1} \pi^{-1}(k) h_{0}, l_{0}\right\rangle\left\langle\pi(k) A_{2} \pi^{-1}(n) h_{0}, l_{0}\right\rangle, \\
& =\sum_{n}\left\langle A_{1} e_{m}, e_{k}\right\rangle\left\langle A_{2} e_{k}, e_{n}\right\rangle, \\
& =\sum_{n} \sigma_{A_{1}}(m, n) \sigma_{A_{2}}(n, k) .
\end{aligned}
$$

Finally, is there a relationship between the covariant and contravariant symbols of these operators?

Proposition 3.18. If an operator $A$ on $l^{2}(\mathbb{Z})$ has a contravariant symbol a $(m)$ which satisfies (3.6), then the covariant symbol of A which satisfies (3.4) is

$$
\tilde{a}(m)=a(m)
$$

Proof. Let an operator $A$ on $l^{2}(\mathbb{Z})$ have a contravariant symbol $a(m)$ which satisfies (3.6), that is

$$
A=\sum_{k} a(k) p_{k}
$$

Then

$$
A e_{m}=\sum_{k} a(k) p_{k} e_{m}=\sum_{k} a(k)\left\langle e_{m}, e_{k}\right\rangle e_{k}=a(k) e_{k}
$$

Now by (3.4)

$$
\begin{aligned}
\tilde{a}(m) & =\left\langle A e_{m}, e_{m}\right\rangle=\left\langle a(k) e_{k}, e_{m}\right\rangle \\
& =\sum_{n} a(k) e_{k}(n) \bar{e}_{m}(n)=a(m)
\end{aligned}
$$


Proposition 3.19. If an operator $A$ on $l^{2}(\mathbb{Z})$ has a contravariant symbol a $(m, n)$ which satisfies (3.7), then the covariant symbol of A which satisfies (3.5) is

$$
\tilde{a}(m, n)=a(n, m) .
$$

Proof. Let the operator $A$ on $l^{2}(\mathbb{Z})$ have a contravariant symbol $a(m, n)$ which satisfies (3.7), that is

$$
A=\sum_{j} \sum_{i} a(i, j) p_{(i, j)}
$$

Then

$$
A e_{n}=\sum_{j} \sum_{i} a(i, j) p_{(i, j)} e_{n}=\sum_{j} \sum_{i} a(i, j)\left\langle e_{n}, e_{i}\right\rangle e_{j}=\sum_{j} a(n, j) e_{j}
$$

Now by (3.5)

$$
\begin{aligned}
\tilde{a}(m, n) & =\left\langle A e_{n}, e_{m}\right\rangle=\left\langle\sum_{j} a(n, j) e_{j}, e_{m}\right\rangle=\sum_{k} \sum_{j} a(n, j) e_{j}(k) \bar{e}_{m}(k) \\
& =\sum_{k} a(n, k) e_{k}(m)=a(n, m) .
\end{aligned}
$$

\section{Conclusion}

In this paper, we introduced the concepts of covariant and contravariant symbols of operators which generated by a representation of the integer group $\mathbb{Z}$. Then we described some properties of covariant and contravariant symbols in $B\left(l_{2}(\mathbb{Z})\right)$. Also, we reformulated some know results on (existence, uniqueness, boundedness and compactness) of these operators in terms of wavelet transform (covariant and contravariant symbols). Finally, the full investigation to find similar conditions for bounded and compact in term of covariant and contravariant symbols generated by another groups is left for further work.

\section{Acknowledgement}

I am grateful to Dr. Vladimir. V. Kisil for reading the manuscript, correcting errors and valuable hints.

\section{References}

[1] F. A. Berezin, Covariant and contravariant symbols of operators, Izv. Akad. Nauk SSSR Ser. Mat., 36 (1972), $1134-1167$.

[2] F. A. Berezin, Wick and anti-Wick symbols of operators, Mat. Sb. (N.S)., 86 (128) (1971), 578-610.

[3] F. A. Berezine, Method of Second Quantization, Nauka, Moscow, 1988.

[4] F. A. Berezin, General concept of quantization, Comm. Math. Phys., 40 (1975), 153-174

[5] V. V. Kisil, Calculus of operators: covariant transform and relative convolutions, Banach J. Math. Anal., 8 (2) (2014), 156-184.

[6] V. V. Kisil, Wavelets in Banach spaces, Acta Appl. Math., 59 (1) (1999), 79-109.

[7] A. V. Balakrishnan, Applied Functional Analysis, volume 3 of Applications of Mathematics, Springer-Verlag, New York, second edition, 1981.

[8] P. A. Fillmore, J. P. Williams, On operator ranges, Advances in Math., 7 (1971), 254-218.

[9] I. Gohberg, S. Goldberg, M. A. Kaashoek, Basic classes of linear operators, Birkh auser Verlag, Basel, 2003.

[10] A. W. Naylor, G. R. Sell, Linear operator theory in engineering and science, volume 40 of Applied Mathematical Sciences, Springer-Verlag, New York, second edition, 1982

[11] H. Fuhr, Abstract Harmonic Analysis of Continuous Wavelet Transforms, Springer-Verlag Berlin Heidelberg, 2005.

[12] L. Debnath, P. Mikusinski, Introduction to Hilbert Spaces with Applications, Academic Press, Boston, $2,1999$.

[13] V. V. Kisil, Erlangen Programme at Large: An Overview, In S. V. Rogosin and A. A. Koroleva (eds.) Advances in applied analysis, Birkhäuser Verlag, Basel, 2012, pp. 1-94.

[14] V. V. Kisil, Integral representations and coherent states, Bull. Belg. Math. Soc. Simon Stevin., 2 (5) (1995), 529-540.

[15] S. T. Ali, J. P. Antoine, J. P. Gazeau, Coherent States, Wavelets and Their Generalizations, Graduate Texts in Contemporary Physics. Springer-Verlag, New York, 2000

[16] A. Perelomov, Generalized Coherent States and Their Applications. Texts and Monographs in Physics, Springer-Verlag, Berlin, 1986.

[17] F. A. Berezin, M. A. Shubin, The Schrodinger Equation, volume 66 of Mathematics and its Applications (Soviet Series). Kluwer Academic Publishers Group, Dordrecht, 1991.

[18] V. V. Kisil, Covariant transforms, Journal of Physics: Conference Series., 284 (1) (2011), 12-38.

[19] V. V. Kisil, Operator covariant transform and local principle, J. Phys. A: Math. Theor., $\mathbf{4 5}$ (2012), 1-10.

[20] M. Garayev, S. Saltan, D. Gundogdu, On the inverse power inequality for the Berezin number of operators, J. Math. Inequal., 12 (4) (2018), 997-1003.

[21] J. R. Retherford, Hilbert Space: Compact Operators and the Trace Theorem, London Math. Soc. Monographs, Cambridge University Press Cambridge, 1993.

[22] N. I. Akhiezer, I. M. Glazman, Theory of Operators in Hilbert Space, Pitman, Boston, I (1981), 254-218.

[23] I. Chalendar, E. Fricain, M. Gürdal, M. T. Karaev, Compactness and Berezin symbols, Acta Sci. Math., 78 (2012), $315-329$.

[24] M. T. Karaev, M. Gurdal, U. Yamanc1, Special operator classes and their properties, Banach J. Math. Anal., 7 (2) (2013), 75-86. 